\title{
Numerical thermal performance of free convection in metal foam heat sinks with fin edges.
}

\author{
Aqeel Mtasher Uglah $^{\dagger}$, Abbas J. Jubear ${ }^{\ddagger}$ \\ Department of Mechanical Engineering College of Engineering-University of Wasit \\ Department of Mechanical Engineering College of Engineering-University of Wasit
}

\begin{abstract}
This study introduces numerical investigation of natural convection in metal foam heat sinks with two types of fin edges namely fillet and sharp edges. Aluminum metal foam of various pore densities (5, $10,20 \mathrm{PPI})$ and sets of porosities (95\% $\leq \varepsilon \geq 71 \%)$ are used in the simulations. The dimensions of the samples were $100 \mathrm{~mm}$ length, $100 \mathrm{~mm}$ height, $10 \mathrm{~mm}$ thickness and the spacing between fins were kept at $8 \mathrm{~mm}$. Then the metal foam strips were installed onto an aluminum base plate $(100 * 100 * 3.5$ $\mathrm{mm}^{3}$ ). The investigation has been performed by control volume method. As well as, the energy equations are established on a principle that based on a local thermal non-equilibrium (LTNE) model in which the temperature of the fluid and the solid matrix are solved separately. The heat input was different from 4 to $30 \mathrm{~W}$ in numerical runs and the air was used as a working fluid. The results show that the base temperature of the heat sink is lower for the new design of edges. Therefore, the proposed approach of the heat sink has significant potential to be utilized to enhance the thermal performance of heat sinks and thereby to develop more advanced effective cooling devices and techniques. The maximum enhancement of the heat transfer coefficient with fillet profile reached $5.6 \%$ greater than fins with sharp edges.
\end{abstract}

Keywords: Natural convection, Fins edges, Metal foam, Heat sink, CFD

\section{Introduction}

Thermal management represents one of the main issues in the electronic packaging processes because high heating causes many problems notably that it reduces the life of electronic components and weaken their reliability unless there are ways to control it.For example cooling for LED (Light Emitting Diode), which is supposed to not exceed the operating temperature of (100 $110)^{\circ} \mathrm{C}[1]$. Although many advanced cooling methods are suggested as liquid cooling or cooling by two -phases, the thermal management of the electronic components depends largely on the heat sink with the extended surface, or so-called fins, through which the heat source is dispersed. The design of extended surfaces (fins) greatly determines the area of heat transfer and flow specifications within the structure. The perfect structure must have a high fluid-solid interfacial area as well as a high heat transfer coefficient, but low flow resistance. Anyway, the first two factors usually opposed to the last. Therefore, one should design the structure of extended surfaces ideally to balance between the conflicting factors to gain maximum heat transfer under any specific conditions. Typically, modern heat sinks free convection heat transfer use in most applications specific fins structures as extended surfaces. There are a lot of studies interested in this regard and using the extended surfaces in various forms and different, such as parallel continuous fins $[2,3]$, radial continuous fins $[4,5]$, tree-shaped continuous fins [6], pin-fins [7], pin-fins with single hole [8], etc. In addition to all the aforementioned structures of fins, open metal foams have been used as extended surfaces also due to their light weight, high thermal efficiency, high surface-to-volume ratio, excellent fluid mixing. Due to these important advantages, metal foams have become 
attractive to many researchers and have been increasingly used in cooling. Feng et al. [9], investigated experimentally natural convection in copper metal foam heat sinks with spacing. The tested specimens with a fixed pore density of 5 pores per inch and fixed porosity of 0.91 . The dimensions were $100 \mathrm{~L} * 10 \mathrm{~W}$ with various heights $(10 / 20 / 40 / 60 / 80 \mathrm{~mm})$ and open slot width between fins were $(2.86 / 5 / 8 / 12.5 / 20$ $\mathrm{mm})$. The results explained that the optimum open slot width exists ranged (5to8) and the best height at $(80 \mathrm{~mm})$, Hetsroni et al. [10], Investigated experimentally free convection heat transfer in metal foam strips with internal heat generation by using IR technique. Metal foam specimens have two pore densities and porosities (20PPI with 0.90 , 40PPI with 0.85 ). The cross-section area of the tested samples was fixed at $(22 \times 10)$ in $\mathrm{mm}$. The test results showed that the heat transfer rate is improved for larger pore sizes 20PPI (fewer pore densities) due to higher permeability], Schampheleire et al. [11], Investigated free convection in slender metal foams. Two samples with a fixed porosity of $93 \%$ and different pore densities of (10PPI, 20PPI) were tested experimentally. The samples with a length of $25.4 \mathrm{~cm}$ and width $2.54 \mathrm{~cm}$. The heights were varied as $(6 / 12 / 18 / 25.4 / 40) \mathrm{mm}$ and $(6 / 12 / 18) \mathrm{mm}$, of 10 and $20 \mathrm{PPI}$. The results explained that the pore densities have a higher impact on the Nusselt number values, Where the sample of 10 PPI foam gives performance up to $25 \%$ better in heat transfer compared with20 PPI foam for the same dimensions used. The heat transfer rate decreases for larger foam height and the best height of the sample was $6 \mathrm{~mm}$. Qu et al. [12], an experimental study in copper metal foam under natural convection. Seven samples with different pore density (10-40 PPI) with $(0.90-0.95)$ porosity and heights from (10 to $50 \mathrm{~mm}$ ) were measured at different inclination angles of $(0,15,30,45,60,75$, and 90). The

\section{Problem Statement}

The reliability of the performance and the life expectancy of the electronic components and equipment are important factors that are closely related to the temperature of any electronic device. The relationship between them is inverse. However, the reduction of the temperature is fully compatible with increasing the reliability and age of the electronic devices. So, control of the temperature of the device through free convection is of vital importance. Therefore, the focus of this study was to find a new design of the heat sink using fins with fillet edges, which proved the ability to cool results also showed that the rate of heat transfer in the metal foams increased with decreases in porosity and pore density, regardless of the directions and heights. The sample with porosity 0.9 , pore density of 10 PPI with an inclination range (60-75) gave the higher average Nssulet number among the studied tested samples. Phanikumar and Mahajan [13], Presented experimental and numerical study of buoyancy-induced flows in high porosity aluminum metal foams. Foam samples with different pore densities (5-40 PPI) and porosity $(0.89-0.97)$ were used in both studies. The size of the samples with 6.35 $\mathrm{cm}$ (Length), $5.08 \mathrm{~cm}$ (Height). The results showed that for a given PPI, the heat transfer coefficient increases with the decrease in porosity due to the dominant role played by conduction improving heat transfer, increasing the permeability (Darcy number) helps the flow to penetrate the foam layer more deeply, thus, contributing to an increased rate of heat transfer. Bhattacharya and Mahajan [14], studied free convection in aluminum metal foams experimentally. The test samples have different pore densities (5, 10, 20, 40 PPI) and different porosity ranges $(0.89-0.96)$. The dimensions of the tested models were fixed at $(6.25 \times 6.25 \times 5) \mathrm{cm}$, (length $\times$ width $\times$ height). The results explained that the samples which have 5 PPI are found to give superior values of heat transfer coefficient (h) when compared to the 20 PPI samples. The main objective of this study is to reduce the base temperature of the heat sink by making a significant change in the shape of the edge of fins using the fillet profile. This change in the shape contributed effectively to reducing the temperature of the base. Since it has not been addressed by all researchers, so this study highlighted in it and achieved outstanding results in terms of thermal performance of the heat sink.

electronic components better than if used with sharp edges.

\section{Numerical Analysis}

The numerical solution comprises a discussion of the following sub-sections: governing equations, computational of the domain, the grid-independence analysis, setting model, setting the cell zone condition, defining boundary conditions. As well as, the validations of current numerical work with another previous numerical study have been discussed. 


\subsection{Computational Domain}

The different components of the model were created by using ANSYS R16.1 software. These components consist of fins, base plate, and a box. These components are individually built up and after then assembled. The fins were arranged in the middle as well as the base on which the fins were mounted and then the enclosure box was constructed. Two types of fin edges have been modeled: fins with fillet edges and fins with sharp edges as shown in figure 1 and figure 2 . All these components are lastly assembled to be ready for the mesh. The

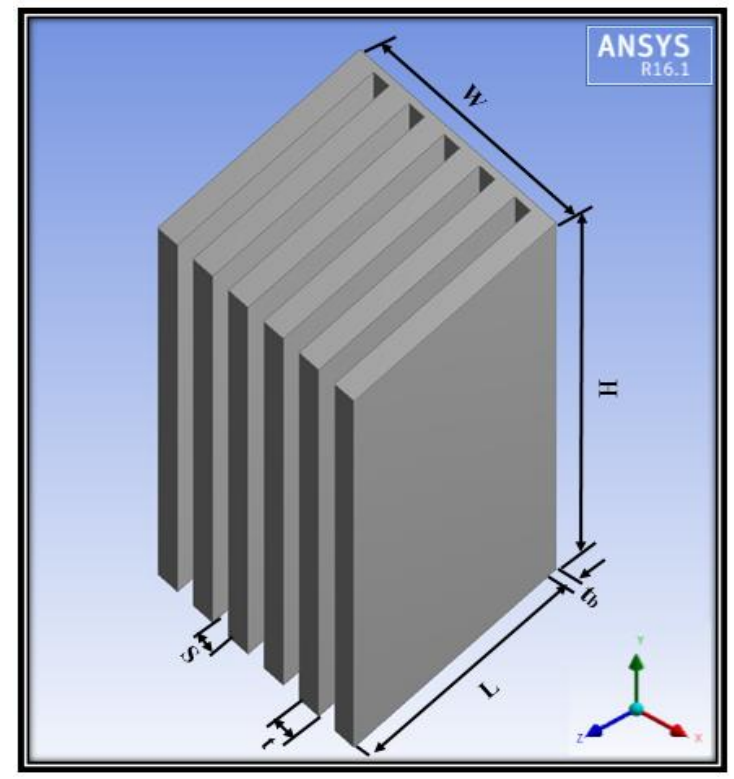

Fig.1: Heat sink with fins of sharp edges

Table 1. Geometrical parameters properties of metal foam and air

\begin{tabular}{|c|c|c|c|c|c|}
\hline \multirow[b]{2}{*}{$\begin{array}{l}\text { Alumi- } \\
\text { num } \\
\text { foam }\end{array}$} & PPI & $\begin{array}{l}\text { Porosity } \\
\qquad(\varepsilon)\end{array}$ & $\begin{array}{c}\mathrm{K}_{\mathrm{s}} \\
(\mathrm{W} / \mathrm{m} . \mathrm{K})\end{array}$ & $\begin{array}{c}\text { Length (L) } \\
\text { mm }\end{array}$ & 100 \\
\hline & $5,10,20$ & $\begin{array}{l}95 \geq \varepsilon \\
\geq 71\end{array}$ & 180 & $\begin{array}{l}\text { Height }(\mathrm{H}) \\
\text { mm }\end{array}$ & 100 \\
\hline \multirow{3}{*}{ Air } & $\mathrm{K}_{f}$ & $\operatorname{Pr}$ & $T_{a m b}$ & $\begin{array}{c}\text { Thickness }(\mathrm{t}) \\
\mathrm{mm}\end{array}$ & 10 \\
\hline & \multirow[t]{2}{*}{0.0265} & \multirow[t]{2}{*}{0.7} & \multirow[t]{2}{*}{$21.5^{\circ} \mathrm{C}$} & $\begin{array}{c}\text { Fin spacing } \\
\text { (s) } \mathrm{mm}\end{array}$ & 8 \\
\hline & & & & $\begin{array}{l}\text { Fillet radius } \\
\text { (r) } \mathrm{mm}\end{array}$ & 2.5 \\
\hline
\end{tabular}

foam fins were fixed on an aluminum base plate of dimension $100 * 100 * 3.5 \mathrm{~mm}$. The boundaries of the process domain were set sufficiently away from the fins to prevent any flow-reversal throughout the numerical analyses. With respect to the selection of the domain dimensions, the optimum fin spacing (s) is calculated experimentally as mentioned in [9] and it has been used in the present work and it was kept at the optimum values $\mathrm{s}=8$ $\mathrm{mm}$. The geometrical dimensions and some of the properties of the metal foam and air that's used in the present work are listed in Table 1.
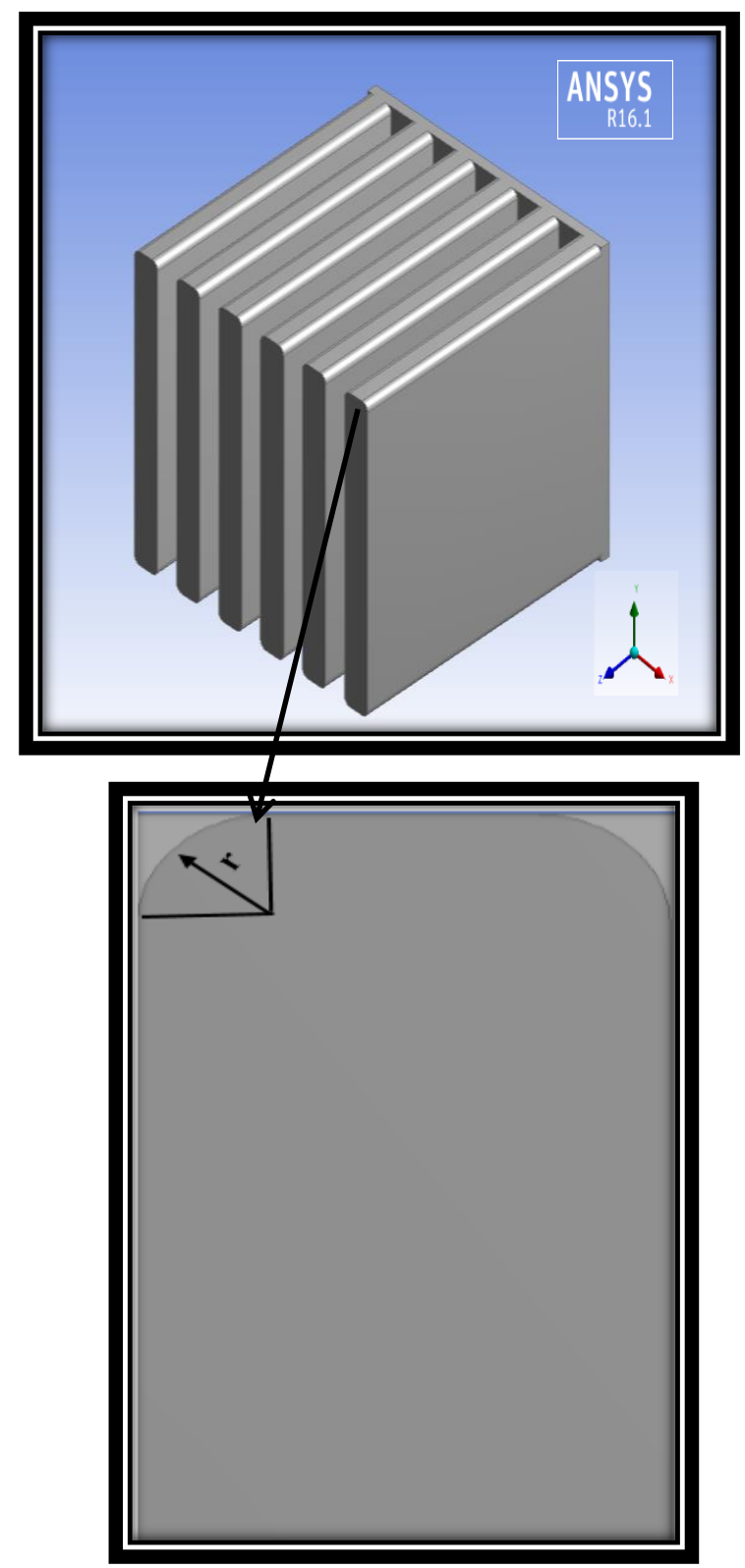

Fig.2: Heat sink with fins of fillet edges 


\subsection{Assumptions}

In order to solve the equations of the flow and heat transfer (continuity, momentum and energy equations), sets of assumptions must be made to simplify the problem.

- Laminar flow.

- Steady state.

- Boussinesq approximation is used.

- Incompressible fluid.

- There is no internal heat generation and neglecting the radiation

- The energy equations are written based on a local thermal nonequilibrium (LTNE) model in which the temperature of the solid matrix and the passing fluid are solved separately.

\subsection{Governing Equations}

The governing equations used in the ANSYS simulation include the momentum equations as well as the continuity and energy equations that belong to porous mediums, especially metal foams [15].

\section{Conservation of Mass}

The principle of continuity is that the input mass of any system must be equal to the mass outside it through the surface of the control volume. The expression of this is mathematically for non-compressible fluid as follows:

It can use the approximations until the changing in density becomes small. These $\frac{\delta \mathrm{Vx}}{\delta x}+\frac{\delta V y}{\delta y}+\frac{\delta V z}{\delta z}=0$

Where : $\mathrm{V}_{\mathrm{x}}, \mathrm{V}_{\mathrm{y}}$, and $\mathrm{V}_{\mathrm{z}}$ are velocity component in the direction $\mathrm{x}, \mathrm{y}$, and $\mathrm{z}$ respectively.

\section{Momentum Equations}

The other principles governing fluid flow are derived from Newton's second law, called the Navier-Stokes equations (the conservation of momentum). And with respect to incompressible fluids the full instantaneous equations are taken in this form: when the gravity is in $-\mathrm{y}$ direction, the body force vector $\mathrm{g}=[0,-\mathrm{g}, 0]$. The momentum equations are:

$$
\begin{gathered}
\frac{\rho}{\varepsilon^{2}}\left(V_{x} \frac{\delta V_{x}}{\delta x}+V_{y} \frac{\delta V_{x}}{\delta y}+V_{z} \frac{\delta V_{x}}{\delta z}\right)=-\frac{\delta p}{\delta x}+ \\
\mu_{e}\left(\frac{\delta^{2} V_{x}}{\delta x^{2}}+\frac{\delta^{2} V_{x}}{\delta y^{2}}+\frac{\delta^{2} V_{x}}{\delta z^{2}}\right)-\frac{\mu}{K} V_{x}|\vec{V}|-\frac{\rho F \varepsilon}{\sqrt{K}} V_{x}
\end{gathered}
$$

In X-direction

$$
\begin{aligned}
& \frac{\rho}{\varepsilon^{2}}\left(V_{x} \frac{\delta V_{y}}{\delta x}+V_{y} \frac{\delta V_{y}}{\delta y}+V_{z} \frac{\delta V_{y}}{\delta z}=-\frac{\delta p}{\delta y}+\right. \\
& \mu_{e}\left(\frac{\delta^{2} V_{y}}{\delta x^{2}}+\frac{\delta^{2} V_{y}}{\delta y^{2}}+\frac{\delta^{2} V_{y}}{\delta z^{2}}\right)-\frac{\mu}{K} V_{y}-\frac{\rho F \varepsilon}{\sqrt{K}} V_{y}|\vec{V}| \\
& +\rho g \beta\left(T-T_{\infty}\right)
\end{aligned}
$$

In Y-direction

$$
\begin{aligned}
& \frac{\rho}{\varepsilon^{2}}\left(V_{x} \frac{\delta V_{z}}{\delta x}+V_{y} \frac{\delta V_{z}}{\delta y}+V_{z} \frac{\delta V_{z}}{\delta z}\right)=-\frac{\delta p}{\delta x}+ \\
& \mu_{e}\left(\frac{\delta^{2} V_{z}}{\delta x^{2}}+\frac{\delta^{2} V_{z}}{\delta y^{2}}+\frac{\delta^{2} V_{z}}{\delta z^{2}}\right)-\frac{\mu}{K} V_{z}|\vec{V}|-\frac{\rho F \varepsilon}{\sqrt{ } K} V_{z}
\end{aligned}
$$

In Z-direction

\section{Energy Equation}

$$
\begin{aligned}
& \rho C_{p}\left(V_{x} \frac{\delta T}{\delta x}+V_{y} \frac{\delta T}{\delta y}+V_{z} \frac{\delta T}{\delta z}=k_{e}\left(\frac{\delta^{2} T}{\delta x^{2}}+\right.\right. \\
& \left.\frac{\delta^{2} T}{\delta y^{2}}+\frac{\delta^{2} T}{\delta z^{2}}\right)
\end{aligned}
$$

approximations are also helpful for faster to reach convergence of the simulation. 
The coefficient of the volumetric thermal expansion must be defined, and this parameter represented the variations of the In free convection studies, the condition of the fluid that it located Away from the hot or cold surface is mentioned by the subscript (infinity). This subscript acts as a reminder that this is the value of a certain entity at a specific distance where the presence of the surface is not felt. Equation can rewrite as:

$$
\beta \approx-\frac{1}{\rho} \frac{\Delta \rho}{\Delta T}=-\frac{1}{\rho}\left(\frac{\rho \infty-\rho}{T \infty-T}\right) \quad \text { At constant } \mathrm{p}
$$

\subsection{Mesh Generation}

FLUENT software is a finite volume numerical method. The solution by numerical methods requires meshing for the geometry. The accuracy of the solution depends on the size of the meshes (cells). The small mesh size introduces more accuracy solution, but whenever the mesh size decreases the number of meshes increase. The result is that the capacity of the computer is large in addition to the time factor, where increasing the number of cells the researcher needs a lot of time. Therefore, the researcher should provide a compromise for this problem because reducing the size of cells requires a large memory of the computer that is used. In FLUENT software there is two types of grids consist of the tetrahedron or hexahedron cells (or a

Table 2. Overview of mesh models density for the fluid with a temperature at constant pressure: $\beta=-\frac{1}{\rho}\left(\frac{\delta \rho}{\delta T}\right)_{p}$

combination between them) in 3-D. The selection of the mesh depends on the type of the application. For applications that involve the complex geometry, the creation of structure or block-structure grids consisting of hexahedron cells, it can be a waste of time. If not impossible, the time of the setup is considered the main motivation for using unstructured- grids that it contains tetrahedron cells.

\subsection{The Optimum Mesh Size Procedure}

For to investigate the spatial accuracy of mesh, five various meshes were constructed for three-dimensional simulation using ANSYS software (R16.1), as explained in Table 2. After simulation of the models for all abovementioned meshing sizes the maximum temperature is observed. For the two last meshes mesh 4 and mesh 5 , the prediction of the temperatures is observed to be very close in contrary to the other three kinds. Thereby the mesh M4 has been chosen for the simulation, which it is suitable, adequate for capturing the flow properties inside the domain and the fins as shown in figure (3).

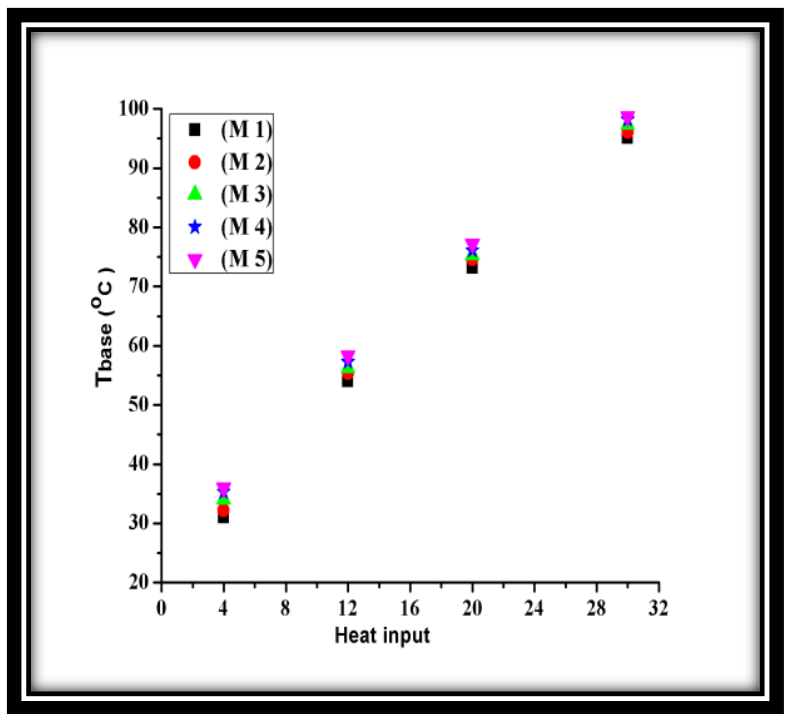


Fig.4a: Boundary condition for domain

\subsection{Boundary Conditions}

Fig.3: Mesh independent of numerical model

In the beginning, flow conditions and other thermal variables are determined on all aspects of the mathematical model being simulated. The identification of these conditions is an important part of the simulation process so it is necessary to identify them appropriately as shown in figure $(4 a, 4 b)$. The boundary conditions that it is applied in this study, the boundary condition applied :

1. At the entrance of the enclosure is the inlet velocity, which is zero at the bottom of the fin array.

2. With respect to the top of the enclosure, the pressure outlet is applied at the outlet of the fin array as a boundary condition.

3. The setting of other sides of an enclosure is set as pressure outlet also, this meaning the foam fins are exposed to the effect of the atmospheric pressure.

4. For the type of flow has been applied as a laminar flow in numerical simulation of this study.

5. There are various values of power input (4, $8,12,16,20,24$ and $30 \mathrm{~W}$ ) have been used in the present work.

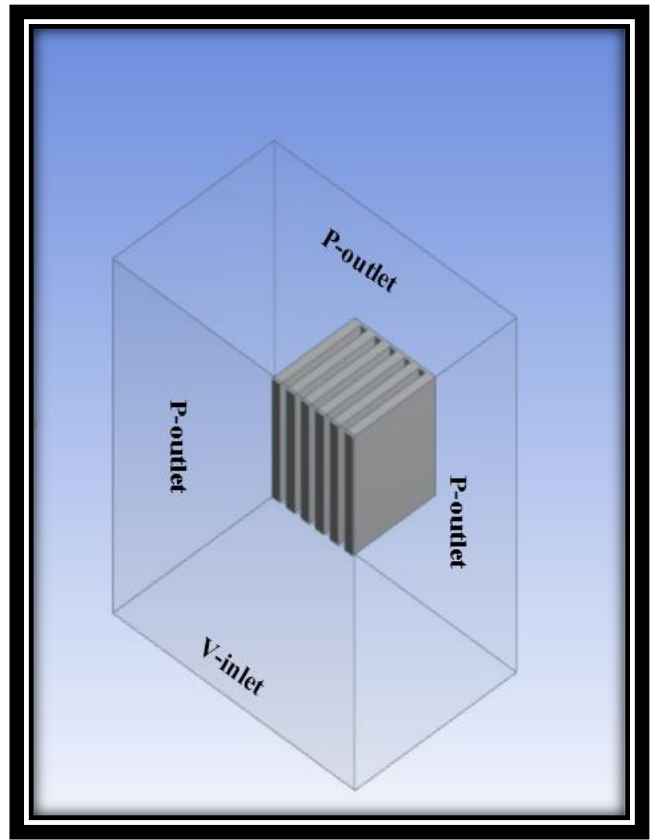

6. A very soft mesh is used especially in areas near the fin surface to capture flow behavior with extreme accuracy.

7. The major condition of the fins model at the base plate is a constant heat flux.

8. There are two kinds of heat transfer occur, the first one: in the fin base is transferred by conduction and the second at the surface of the fins is carried out by convection.

9. The area around the base of the fins is defined as isolated

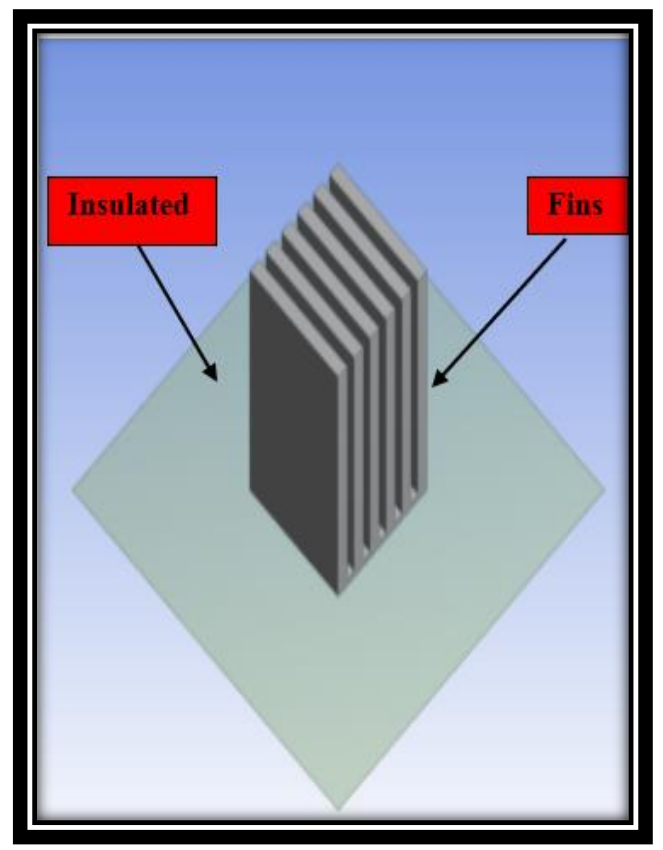

Fig.4b: Boundary condition for domain

\section{. Results and Discussions}

\subsection{Validation of the present work}

In order to validate the results of the numerical simulation of the current work, a comparison was made with Feng et al., 2017 [9]. It can be seen from figure 5 is that there is an excellent agreement between the validity of our numerical results and his experimental results, where the error rate does not exceed $5 \%$. This validation has been performed 
for copper foam fins with 5 PPI, porosity $91 \%$ and dimensions of $100 \mathrm{~mm}$ length $* 100 \mathrm{~mm}$ height $* 10$ $\mathrm{mm}$ thickness as well as, the space between fins was $8 \mathrm{~mm}$.
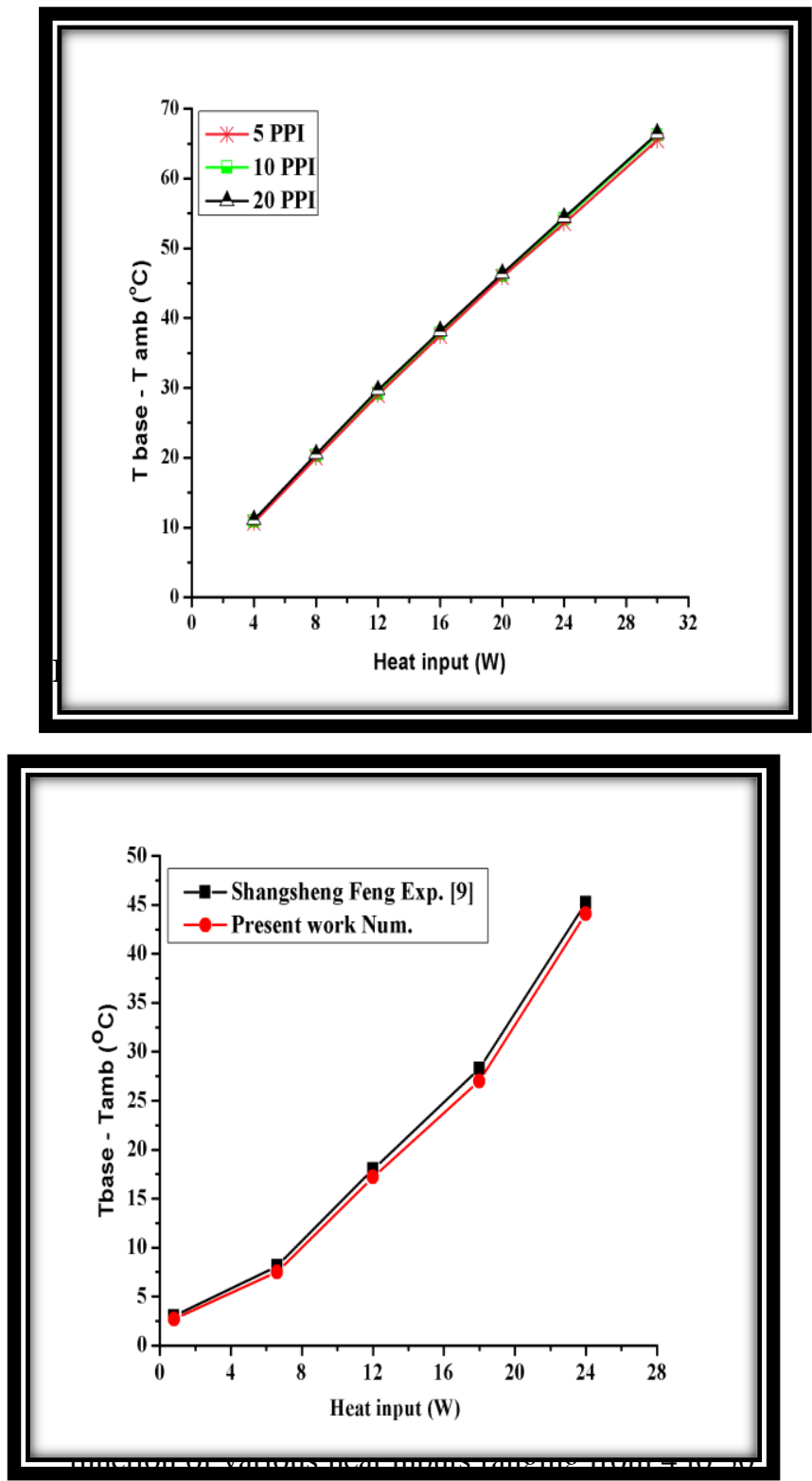

W. Through the curves that were drawn, it is clear the relation between average temperature difference and heat input is positive. In another meaning, the 4.3 Effect of pore density on heat transfer coefficient

The variations heat transfer coefficients are plotted with various heat input. The effecting of pores per inch on the heat transfer coefficients can be explained by making a comparison between three different types of pore densities namely $(5,10$, and $20 \mathrm{PPI}$ ), and it have porosity equal 79\%. By looking the figure (7), it appears that there is a

positive relationship between heat transfer coefficients and heat input, where the increase in increasing of heat input leads to increase in the temperature difference directly. Pore size or

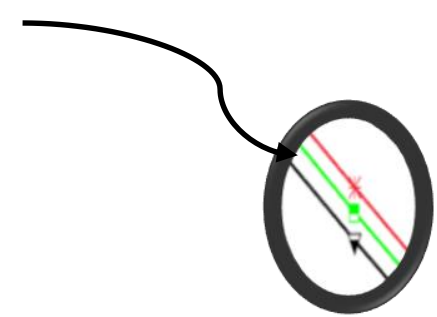

Fig. 6: Variation of average base temperature to ambient with heat input of different pore densities

(density) is considered one of the most factors that it is impact in different ways on the heat transferinside the metal foams. At the same value of porosity, the average temperature difference increases when the pore size increases also. This is in fact, due to that the flow resistance reduces with an increasing in pore size (or a decreasing in porety) resulting in enhanced of flow mixing and heat transfer through an internal structure of metal foams. Therefore, the metal foams with pore density 5 PPI showed a slight enhancement in reducing the average base temperature foam sample with pore density 10 PPI see table 3 .

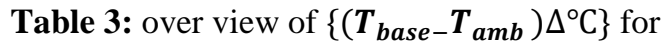
$5,10,20 \mathrm{PPI}$ and porosity $79 \%$

\begin{tabular}{|c|c|c|c|}
\hline $\begin{array}{c}\text { Heat } \\
\text { input } \\
\text { (W) }\end{array}$ & $\mathbf{1 0}$ PPI & $\mathbf{5}$ PPI & 20 PPI \\
\hline 4 & $11.1 \Delta^{\circ} \mathrm{C}$ & $10.7 \Delta^{\circ} \mathrm{C}$ & $10.9 \Delta^{\circ} \mathrm{C}$ \\
\hline 8 & $20.5 \Delta^{\circ} \mathrm{C}$ & $20 \Delta^{\circ} \mathrm{C}$ & $20.3 \Delta^{\circ} \mathrm{C}$ \\
\hline 12 & $29.7 \Delta^{\circ} \mathrm{C}$ & $29 \Delta^{\circ} \mathrm{C}$ & $29.2 \Delta^{\circ} \mathrm{C}$ \\
\hline 16 & $38.13 \Delta^{\circ} \mathrm{C}$ & $37.5 \Delta^{\circ} \mathrm{C}$ & $37.8 \Delta^{\circ} \mathrm{C}$ \\
\hline 20 & $46.35 \Delta^{\circ} \mathrm{C}$ & $45.9 \Delta^{\circ} \mathrm{C}$ & $46.1 \Delta^{\circ} \mathrm{C}$ \\
\hline 24 & $54.4 \Delta^{\circ} \mathrm{C}$ & $53.6 \Delta^{\circ} \mathrm{C}$ & $54.2 \Delta^{\circ} \mathrm{C}$ \\
\hline 30 & $66.45 \Delta^{\circ} \mathrm{C}$ & $65.5 \Delta^{\circ} \mathrm{C}$ & $66.2 \Delta^{\circ} \mathrm{C}$ \\
\hline
\end{tabular}

heat input results in increased heat transfer coefficients as well. Pore density is one of the important design factors that contribute significantly to the description of the internal structure of metal foams. The heat transfer coefficient increases when the pore densities decrease as shown in the figure. It is noted that flow resistance is closely related to pore density as the flow resistance decreases with increased pore size due to this reason, it happens enhancement in heat transfer because of good flow mixing of fluids that it passes through metal foam. As result, the metal 
foam which has 5 PPI shows a slight average improvement in heat transfer coefficients estimated about $1.3 \%$ greater than the foam of 10 PPI which has an average of improvement $1 \%$ superior to the foam with 20 PPI.

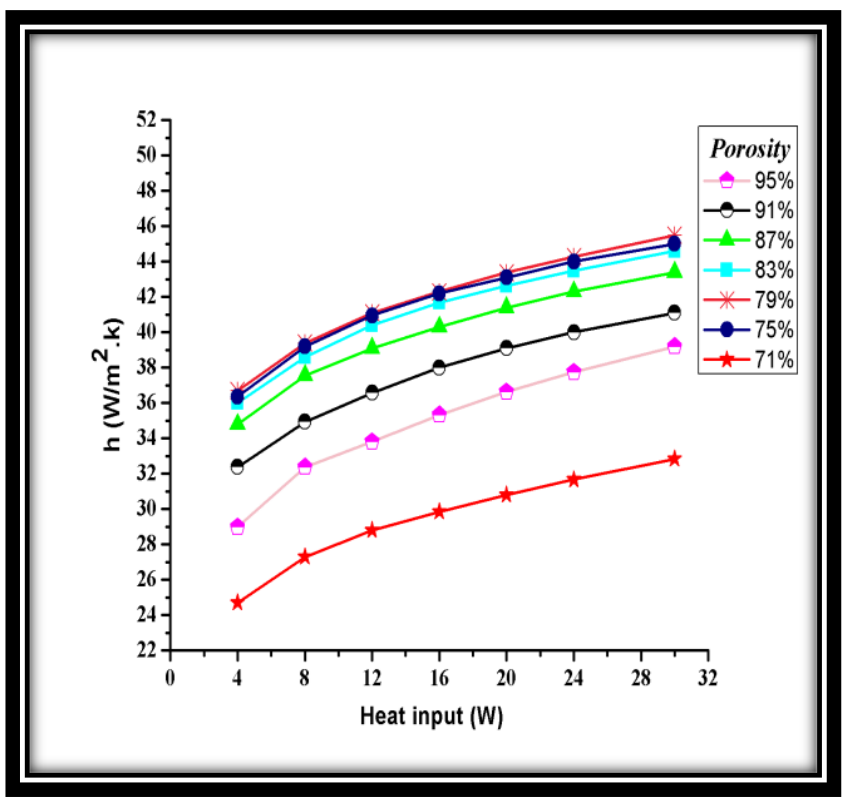

Fig. 7: Variation of heat transfer coefficient as a function of heat input of various pore densities structure of the metal foam occurs. As shown in graph, the average temperature difference starts to drop gradually from porosity $95 \%$ to porosity $79 \%$. After that, the inversion point of enhancement of thermal performance take places at porosity of $71 \%$.

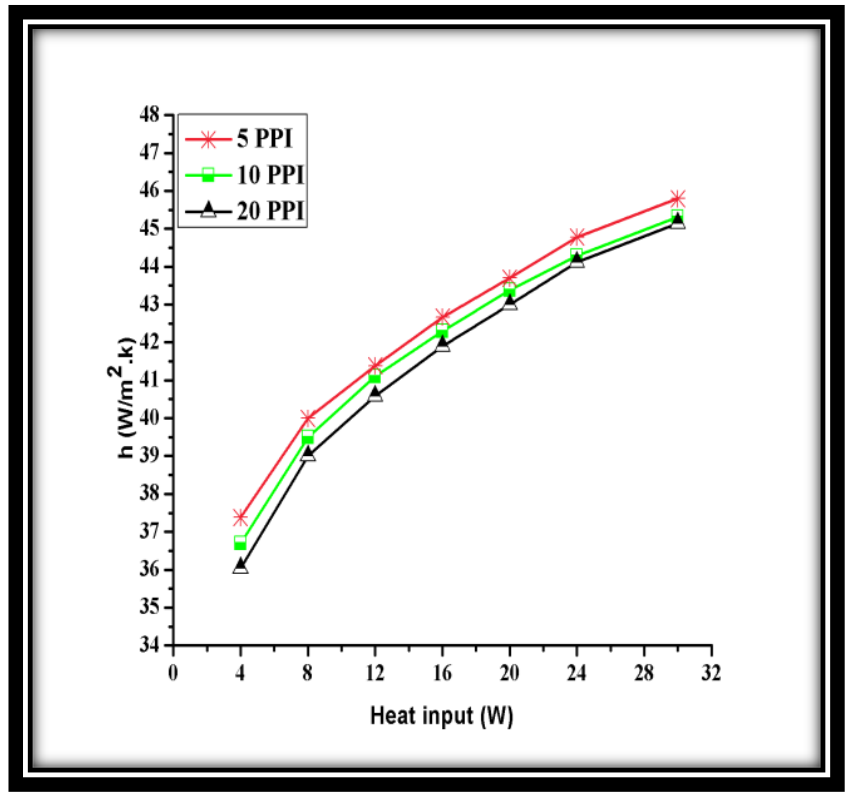

Fig. 8: Variation of average base temperature to ambient with heat input of different porosities.

\subsection{The influence of porosity on the heat transfer coefficient}

The heat transfer coefficients are drawn with different values of the heat input ranged of (4 to 30 $\mathrm{W})$ as shown in the figure (9). The porosities varied of $(0.95 \leq \varepsilon \geq 0.71)$ it can be noted from the figure that for a constant value of pore density(PPI), the heat transfer coefficients increase with a decreasing in porosity value. As the porosity decreases, the thickness of fibers to be large, thereby is more metal content per unit volume, as result, the heat transfer by conduction through the metal fibers contributes to an additional increase in the heat transfer. The highest rate of enhancement in heat transfer coefficient with the porosity of 0.79 compared to 0.71 , it reaches up to $32.7 \%$. This is because the porosity of 0.79 has the lowest temperature difference; as a result, the highest values of heat transfer coefficient are its share. 


\subsection{The influence of edge type of the fins on base temperature}

The average temperature difference is drawing with various heat input for fixed porosity of $91 \%$ with two types of edges, namely fillet and sharp as shown in figure (10). As expected, the average temperature difference is increased when the heat input increase. It can be noted that for the same dimensions of the heat sink, the fins with the fillet edge have the average temperature difference lesser than the sharp edge fins. This excellence in thermal performance is due to the reduction of the base temperature because the fillet profile is contributing significantly to eject a large amount of heat from the hot region. This is because the fins design by these configurations helps the air to penetrate between the fins with more freedom and smoothness. Thereby, the thermal performance of the fins with a new design will be much better than fins with sharp edges. Also, the actual improvement begins from heat input $20 \mathrm{~W}$ as shown below. As well as the average of improvement in the base temperature of the heat sink with the fillet profile was about $4 \%$ in comparison to the heat sink with sharp edges.

\subsection{Effect of edge type of the fins on heat transfer coefficient}

The heat transfer coefficient plot as a function of various heat input to know the effecting of fillet profile and a sharp edge on improving heat transfer coefficient for metal foam heat sinks as shown in figure (11). It is clear that when the values of the heat input are high, this means that the heat transfer coefficients are high also, that is a note in the figure. The heat sinks designed with fillet profile have the highest heat transfer coefficients compared to the heat sinks with a sharp edge. This can be explained briefly because the new design of heat sink has the latest base temperature to ambient due to the increased amount of air that it touches the fins. Thus, the amount of heat which has been removed is greater. The new structure of the heat sink has minimized the zone of collision between the rising air as a result, of buoyancy effect and the fins.

\subsection{Temperature Contours}

In order to enhance previous understandings and distancing the contribution of addition a fillet edges for the metal foam fins on the overall thermal enhancement of a new design of heat sink, finned metal foam heat sinks with and without fillet profile are compared as shown in figure (12).

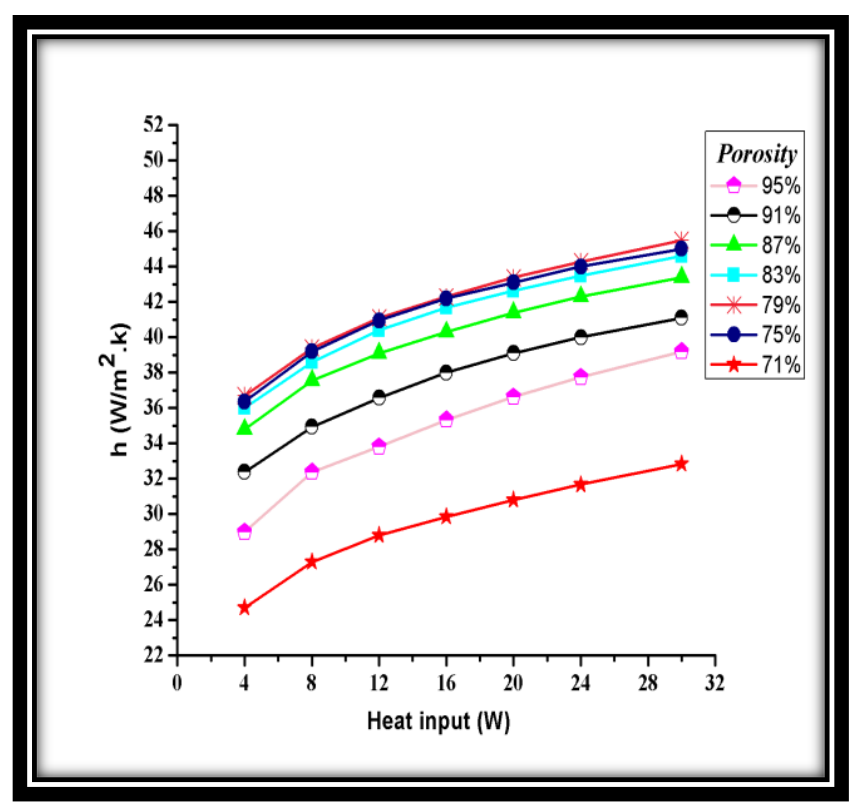

Fig. 9: Variation of heat transfer coefficient as a function of heat input of various porosities

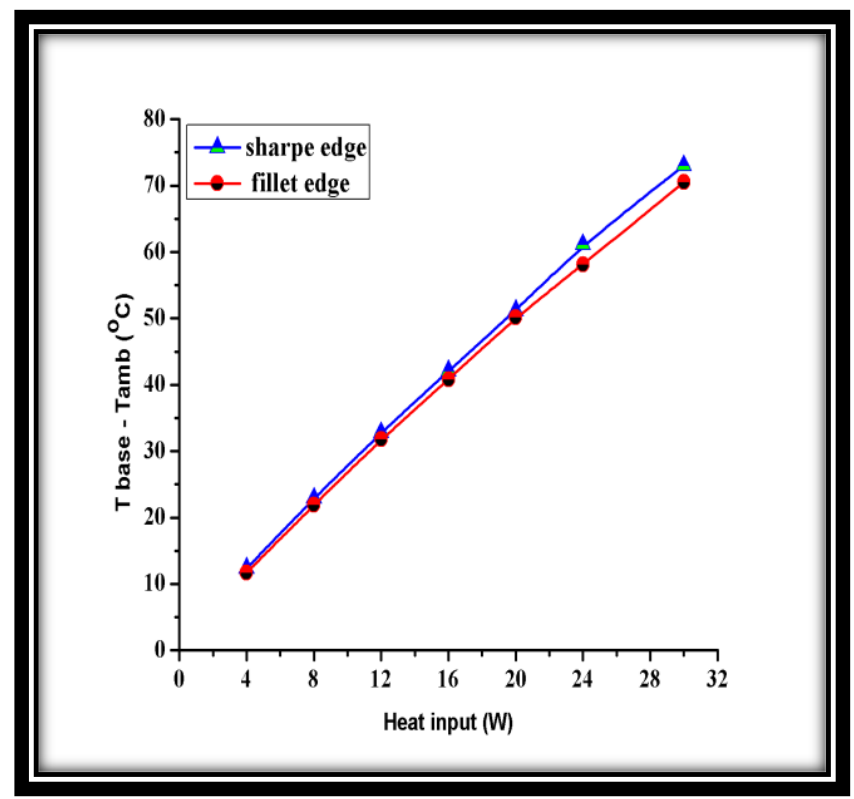

Fig. 10: Variation of average base temperature to ambient with heat input for two types of fin edges

To determine the effect of porosity on the base temperature was compared between two samples of same PPI and at the constant heat input, it was observed that by reducing the value of porosity, the difference in temperature is also reduced and this gives a good indicator of enhancing in the thermal 
performance of the heat sink, as shown in figure

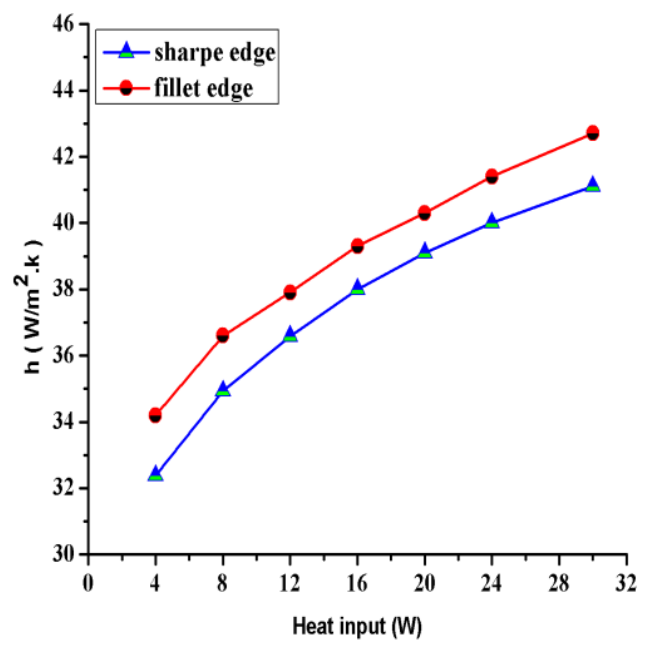

Fig. 11: Variation of heat transfer coefficient with

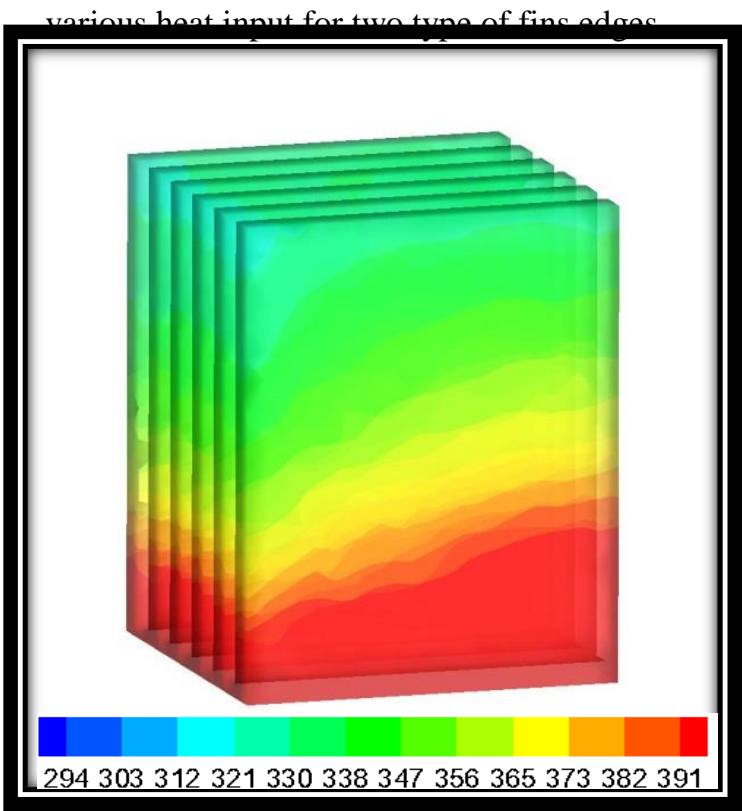

(a) Foam with Porosity of $79 \%$
(13).

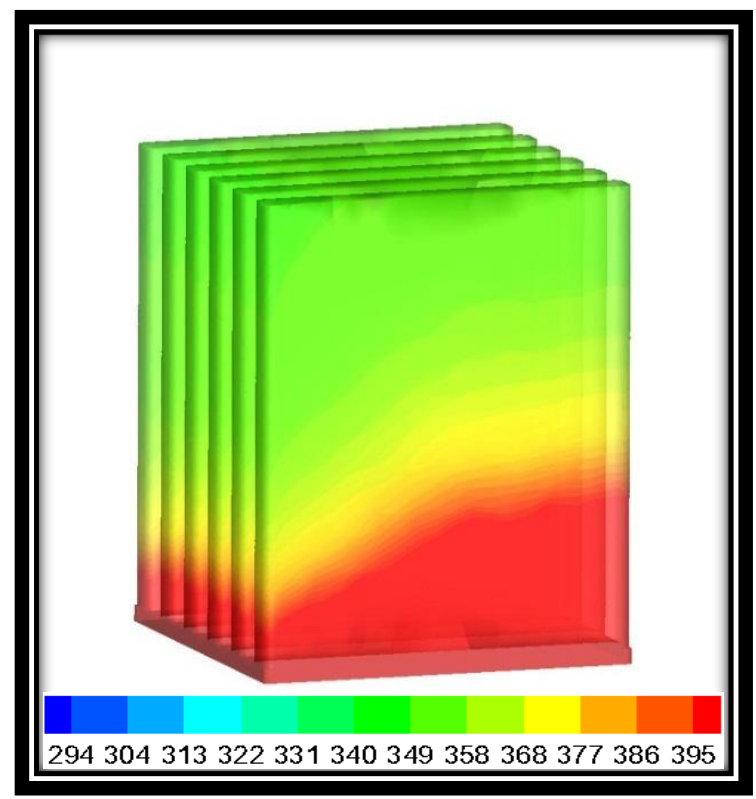

(a) heat sink with fillet edges

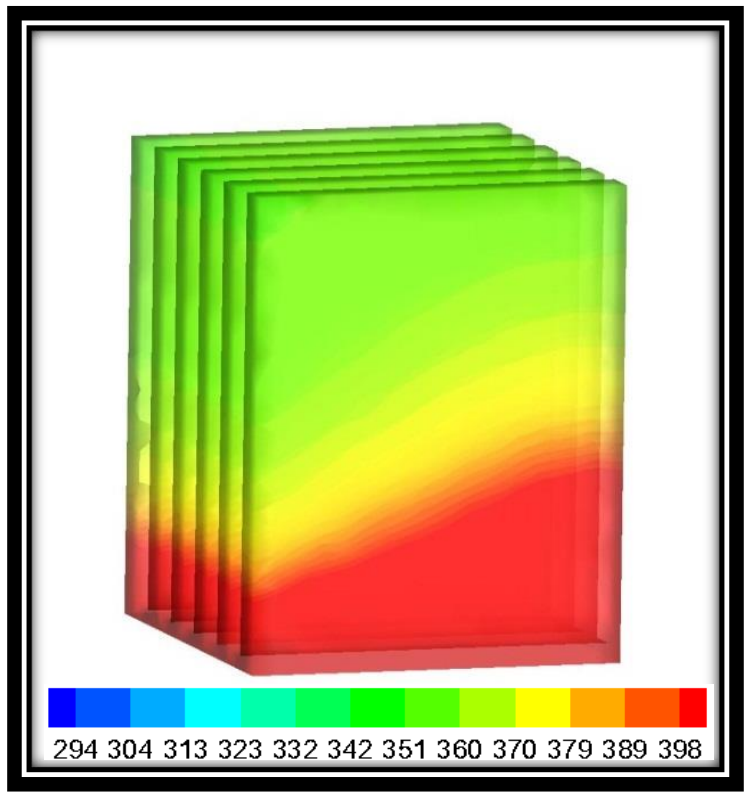

(b) heat sink with sharp edges

Fig. 12. Contour temperature (k) of the heat sink with and without fillet profile at $30 \mathrm{~W}, 10 \mathrm{PPI}$

\section{4-9 Correlation equation}

The figure (14) shows both predicted and numerical values of Nusselt number. It can be seen that the model generally conducts a good prediction of the Nusselt number change over different values of porosity. The average goodness of fit of the model

was about 90\%. The relation which gives the minimum error for experimental and predicted 
rustles. The developed empirical equation has the form as in the following equation. result of this study is that the modern design of the heat sink with fillet fins has achieved a remarkable improvement in thermal performance compared to the old design of the heat sink,

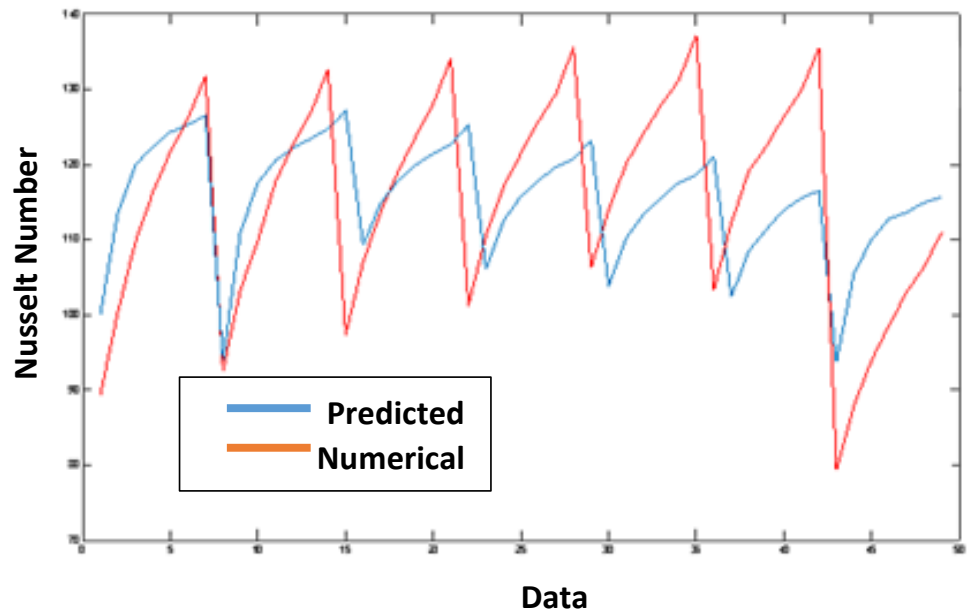

Fig. 14. The relation between numerical and predicted data.

$N_{u}=C_{1}+C_{2} *(\epsilon)^{C_{3}}+C_{4} * R a^{C_{5}}$

The minimum error is achieved in the equation below

$\%$ error $=\left|\frac{N u_{p}-N u_{\mathrm{exp}}}{N u_{p}}\right| * 100$

$\mathrm{Nu}_{\mathrm{p}}=$ predicted value of Nusselt number.

$\mathrm{Nu}_{\text {exp }}=$ Numerical value of Nusselt number.

$\mathrm{C}_{1}=154.33, \mathrm{C}_{2}=-19393.13, \mathrm{C}_{3}=-1.5$,

$\mathrm{C}_{4}=5,017^{*} 10^{13}$ and $\mathrm{C}_{5}=-1.1$

\section{Conclusion}

Effect two types of fins edges on thermal performance for the metal foam heat sinks under natural convection has been carried out numerically. The comparison was performed by using ANSYS (16.1) software between heat sink with fillet profile (new design) and heat sink with sharp profile (so named conventional design).Different samples with various pores per inch (PPI) and sets of porosities was investigated based on local thermal non-equilibrium (LTNE) model. The obtained results demonstrated that the best pore density for this study is 5 PPI and the optimum porosity which can give lowest base temperature to ambient is $79 \%$. The most important

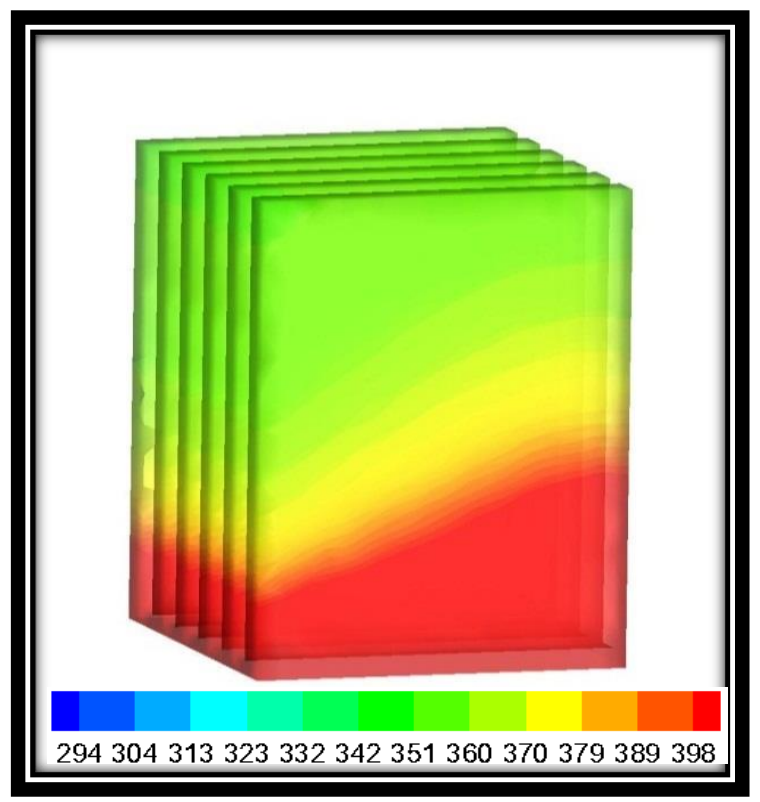

(b) Foam with Porosity of $91 \%$

Fig. 13. Contour temperature $(\mathrm{k})(\mathrm{a}, \mathrm{b})$ of the heat sink with two type of porosities, at $30 \mathrm{~W}, 10 \mathrm{PPI}$.

where, the maximum enhancement of heat transfer coefficient with fillet profile reached $5.6 \%$ greater than fins with sharp edges. As well as the average of improvement in the base temperature of the heat sink with the fillet profile was about $4 \%$ in comparison to the heat sink with sharp edges.

\section{References}

[1] Petroski, J., 2014, Advanced natural convection cooling designs for light-emitting diode bulb systems, Journal of Electronic Packaging, 136(4), pp. 041005.

[2] Shen, Q., Sun, D., Xu, Y., Jin, T., Zhao, X., 2014, Orientation effects on natural convection heat dissipation of rectangular fin heat sinks mounted on LEDs, International Journal of Heat and Mass Transfer, 75, pp. 462-469.

[3] Mehrtash, M., Tari, I., 2013, a correlation for natural convection heat transfer from inclined platefinned heat sinks, Applied Thermal Engineering, 51(1), pp. 1067-1075.

[4] Li, B., Baik, Y.J., Byon, C., 2016, Enhanced natural convection heat transfer of a chimney-based 
radial heat sink, Energy Conversion and Management, 108, pp. 422-428.

[5] Costa, V.A., Lopes, A.M., 2014, Improved radial heat sink for led lamp cooling, Applied Thermal Engineering, 70(1), pp. 131-138.

[6] Almogbel, M.A., 2005, constructed tree-shaped fins, International Journal of Thermal Sciences, 44(4), pp. 342-348.

[7] Yu, E., Joshi, Y., 2002, Heat transfer enhancement from enclosed discrete components using pin-fin heat sinks, International Journal of Heat and Mass Transfer, 45(25), pp. 4957-4966.

[8] Elshafei, E., 2010, Natural convection heat transfer from a heat sink with hollow/perforated circular pin fins, Energy, 35(7), pp. 2870-2877.

[9] Shangsheng Feng, Feichen Li, Fenghui Zhang, Tian Jian Lu.,2017, Natural Convection in Metal Foam Heat Sinks with Open Slots, Experimental Thermal and Fluid Science,47(19)pp. [10] Hetsroni, G., Gurevich, M., Rozenblit, R., 2008, Natural convection in metal foam strips with internal heat generation, Experimental Thermal and Fluid Science, 32(8), pp. 1740-1747.

[11] Schampheleire, S.D., Jaeger, P.D., Reynders, R., et al., 2013, Experimental study of buoyancydriven flow in open-cell aluminum foam heat sinks, Applied Thermal Engineering, 59(1), pp. 30-40.

[12] Qu, Z.G., Wang, T.S., Tao, W.Q., Lu, T.J., 2012, Experimental study of air natural convection on metallic foam-sintered plate, International Journal of Heat and Fluid Flow, 38, pp. 126-132.

[13] Phanikumar, M., Mahajan, R., 2002, NonDarcy natural convection in high porosity metal foams, International Journal of Heat and Mass Transfer, 45(18), pp. 3781-3793.

[14] Bhattacharya, A., Mahajan, R., 2006, Metal foam and finned metal foam heat sinks for electronics cooling in buoyancy-induced convection, Journal of Electronic Packaging, 128(3), pp. 259-266.

[15] M. A. Nim and A. H. Hajeej, "Numerical Study of Mixed Convection Heat Transfer Enhancement in a Horizontal " vol. 17, no. July, pp. 223-241, 2017. 